\title{
Dual roles of Q0A-8a in antiosteoporosis: a combination of bone anabolic and anti-resorptive effects
}

\author{
Jing WU ${ }^{1,2}$, Qi SHEN ${ }^{1}$, Wei CUI ${ }^{1}$, Yu ZHAO ${ }^{1}$, Yue HUAI ${ }^{1}$, Yu-chao ZHANG ${ }^{1}$, Bei-hua BAO ${ }^{1}$, Chen-xi LIU ${ }^{1}$, Qing JIANG ${ }^{2}$, Jian-xin \\ $\mathrm{LI}^{1, *}$ \\ ${ }^{1}$ State Key Laboratory of Analytical Chemistry for Life Science and Collaborative Innovation Center of Chemistry for Life Sciences, \\ School of Chemistry and Chemical Engineering, Nanjing University, Nanjing 210023, China; ${ }^{2}$ Medical School of Nanjing University, \\ Nanjing 210093, China
}

\begin{abstract}
Osteoporotic treatments have largely depended on antiresorptive or anabolic drugs; but the former also suppresses new bone formation, and the latter only includes human parathyroid hormone. There is no drug that has a dual effect to inhibit bone resorption and to stimulate bone formation simultaneously. Here, we report a small molecule, a quinoxaline derivative of oleanolic acid (QOA-8a) that plays such dual roles in osteoblasts and osteoclasts in the treatment of osteoporosis. Osteoclast differentiation was induced by incubation of primary mouse bone marrow-derived macrophages in the presence of RANKL and M-CSF, treatment with QOA-8a dose-dependently inhibited the osteoclast formation with an $\mathrm{IC}_{50}$ value of $0.098 \mu \mathrm{mol} / \mathrm{L}$. QOA-8a also directly acted on osteoblasts, and stimulated new bone formation in murine calvarial bones in vitro and in vivo. In an OVX rat model, administration of QOA-8a (1, $5 \mathrm{mg} \cdot \mathrm{kg}^{-1} \cdot \mathrm{d}^{-1}, \mathrm{po}$ ) for 16 weeks effectively prevented OVX-induced bone loss, accompanied by decreased serum levels of the bone resorption marker CTX-1 and increased serum levels of osteoblast marker N-MID-OT. Meaningfully, our preliminary study revealed that QOA-8a down-regulated the ERK1/2 signal in osteoclasts and up-regulated the signal in osteoblasts. QOA-8a showed dual functions in both animal and human osteoclastogenesis and osteoblastogenesis. Our results demonstrate that QOA-8a might serve as a lead compound with a dual function of bone anabolic and anti-resorptive effects in the development of anti-osteoporosis agents.
\end{abstract}

Keywords: QOA-8a; oleanolic acid; antiosteoporosis; osteoclasts; osteoblasts; OVX rat model; bone mineral density; dual-energy X-ray absorptiometry; microcomputed tomography

Acta Pharmacologica Sinica (2018) 39: 230-242; doi: 10.1038/aps.2017.63; published online 17 Aug 2017

\section{Introduction}

Osteoporosis is a systemic disease characterized by low bone mass and microarchitectural deterioration of bone tissue, with a consequent increase in bone fragility and susceptibility to fracture ${ }^{[1]}$. It is estimated that $30 \%-50 \%$ of women and $15 \%-30 \%$ of men will suffer an osteoporotic fracture in their lifetime, and approximately $80 \%$ of osteoporosis patients are women seen in a clinic setting who present with a dramatic reduction in estrogen levels ${ }^{[2,3]}$. Osteoporosis causes more than 8.9 million fractures annually worldwide and approximately 1000 per hour ${ }^{[4]}$. In 2010, the direct costs of only osteoporotic fractures were estimated at $€ 29$ billion in the five largest EU countries (France, Germany, Italy, Spain and UK) and

\footnotetext{
* To whom correspondence should be addressed. E-mail lijxnju@nju.edu.cn

Received 2017-01-25 Accepted 2017-04-13
}

$€ 38.7$ billion in 27 EU countries ${ }^{[5]}$. By 2025, there will be over 3 million fractures, with related outlays of $\$ 25.3$ billion per year in $\mathrm{USA}^{[6]}$. In China, the yearly expenditure of only osteoporotic hip fracture reaches approximately $\$ 17.4$ billion $^{[7]}$.

Bone remodeling is essential for bone, and the healthy bone is built on a dynamic equilibrium via bone resorption by osteoclasts and bone formation by osteoblasts. A disruption of this equilibrium resulting in excessive bone resorption can lead to osteoporosis. Therefore, therapies for osteoporosis universally act on the coupled system of osteoblast and osteoclast activities $^{[5]}$. Inhibition of functions of osteoclasts and enhancement of osteoblast activities are key therapeutic tools for osteoporosis treatment, and antiosteoporotic treatments have largely depended on antiresorptive or anabolic drugs ${ }^{[8]}$. Currently, the most clinically available therapies are antiresorptive agents, which work by inhibiting the activity of osteoclasts and, accordingly, reducing bone resorption. How- 
ever, osteoclast inhibitors also suppress new bone formation due to the coupling of osteoblast and osteoclast activities ${ }^{[9,10]}$. Anabolic agents act by stimulating formation of new bone, whereas the only clinically available drug is recombinant human parathyroid hormone $(\mathrm{PTH})_{1-34}$ in the US and $\mathrm{PTH}_{1-84}$ in Europe ${ }^{[11]}$. Although PTH stimulates bone formation, the use of rhPTH is also limited to 24 months in the United States and 18 months in Europe due to the increased risk of osteosarcoma $^{[12]}$. Strontium ranelate has been reported to both increase markers of bone formation and decrease markers of bone resorption in vitro ${ }^{[13]}$; however, there is no in vivo evidence to support its bone anabolic efficacy ${ }^{[14,15]}$. Although bisphosphonates (Alendronate) and an antibody to RANKL (Denosumab) can increase bone mass by shutting down cells known as osteoclasts, they also hinder the creation of new bone ${ }^{[16,17]}$. Nevertheless, a combination of teriparatide and denosumab can increase bone mineral density more efficaciously than monotherapy with either agent, especially in postmenopausal women with osteoporosis ${ }^{[18,19]}$. In some ways, these drugs have succeeded, but highly publicized side effects are causing patients to shy away from these bisphosphonates ${ }^{[20]}$ or daily subcutaneous injectable drugs. Therefore, there is a strong medical need to look for new anti-osteoporosis drugs. If an agent could exert a dual effect that increases bone formation and reduces bone resorption simultaneously, it would open a novel window for the treatment of osteoporosis.

Natural products have had a major role as lead structures in drug discovery ${ }^{[21]}$. In our research project targeting new antiosteoporotic agents from natural resources, we screened multiple extracts from traditional Chinese medicine and discovered that oleanolic acid and its glycosides from Achyranthes bidentata Blume (Amaranthaceae) possessed an antiosteoporotic effect ${ }^{[22,23]}$. Further long-term efforts in chemical synthesis and bioassays resulted in a quinoxaline derivative of oleanolic acid (QOA-8a) (Figure 1A) that significantly inhibited the differentiation, formation and bone resorptive activity in osteoclasts without cytotoxicity (Figure $1 \mathrm{~B})^{[24]}$. In ovariectomized mice, QOA-8a prevented bone loss without any hormone-like side effects, and the mice treated with 1 $\mathrm{mg} \cdot \mathrm{kg}^{-1} \cdot \mathrm{d}^{-1}$ maintained the same bone mineral density level as sham mice ${ }^{[25]}$.

In the present study, we found that QOA-8a is capable of elevating alkaline phosphatase (ALP) activity, a marker of osteoblast differentiation in the mouse calvarial osteoblastic cell line MC3T3-E1, which strongly suggested that QOA-8a might have a dual effect by enhancing bone formation and reducing bone resorption. Therefore, the effects of QOA-8a were tested on osteoclast formation using RANKL-induced osteoclast formation assay and on the formation of new bone of murine calvarias both in vitro and in vivo. Then, we used an ovariectomy-induced (OVX) rat model related to postmenopausal osteoporosis to test the dual functions of QOA-8a. Furthermore, a preliminary mechanism of the dual effect was investigated. Finally, the dual effect of QOA8 a was examined in both human-sourced osteoclasts and osteoblasts.

\section{Materials and methods \\ Materials and reagents}

QOA-8a was synthesized by the Key Lab of Analytical Chemistry for Life Science, School of Chemistry and Chemical Engineering, Nanjing University (Nanjing, China), and the purity was more than $99.0 \%$. The other reagents and kits were purchased from the following sources: a modification of Eagle's medium (a-MEM), Dulbecco's modified Eagle's medium (DMEM), Hams F12 (D/F12), fetal bovine serum (FBS), and antibiotic/antimycotic from HyClone (Carlsbad, CA, USA); recombinant murine soluble receptor activator for nuclear factor $\kappa \mathrm{B}$ ligand (m-RANKL), recombinant human soluble receptor activator for nuclear factor $\mathrm{kB}$ ligand (h-RANKL) and recombinant human macrophagecolony stimulating factor (M-CSF) from PeproTech EC (London, UK); penicillin, streptomycin and the TRAP kit from Sigma (St Louis, MO, USA); ALP kit from Jiancheng (Nanjing, China); and BMP-2 kit, OPG kit, N-MID-OT kit and CTX-I kit from R\&D Systems (Minneapolis, USA). Protein expression was examined using antibodies specific to ERK1/2, phosphorylated ERK1/2, p38-MAPK, phosphorylated p38-MAPK, IкBa, phosphorylated IкBa, JNK, phosphorylated JNK, NF-kB-p65, phosphorylated NF-kB-p65, c-Fos, and NFATc1, which were all from Cell Signaling Technology (Danvers, USA).

\section{Cell cultures}

\section{Osteoclast formation assay}

Primary mouse bone marrow monocyte/macrophage cells (BMMs) were isolated from the long bones of 5-week-old male ICR mice following protocols approved by the Institutional Animal Use and Care Committee of Nanjing University. BMMs were plated in a 96-well plate at a density of $3 \times 10^{5}$ cells per well in complete a-MEM medium with 10\% heat-inactivated FBS (penicillin $50 \mathrm{IU} / \mathrm{mL}$ and streptomycin $50 \mu \mathrm{g} / \mathrm{mL}$ ), $30 \mathrm{ng} / \mathrm{mL} \mathrm{M-CSF}$, and $50 \mathrm{ng} / \mathrm{mL}$ RANKL to induce the differentiation of osteoclasts. After 5 days, the cells were stained with the TRAP kit from Sigma (St Louis, MO, USA). TRAP staining was performed as follows: samples were fixed with $4 \%$ paraformaldehyde-PBS, washed with PBS three times and stained with TRAP solution containing $100 \mathrm{mmol} / \mathrm{L}$ sodium acetate buffer ( $\mathrm{pH} 5.0$ ), $50 \mathrm{mmol} / \mathrm{L}$ sodium tartrate, 0.1 $\mathrm{mg} / \mathrm{mL}$ sodium naphtol AS-MX phosphate, $0.6 \mathrm{mg} / \mathrm{mL}$ Fast Violet LB and $0.1 \%$ Triton X-100. Enzymatic reactions were stopped by washing samples with PBS. Osteoclasts were recognized by light microscopy as $>3$ nuclei containing TRAPpositive giant cells. Data are expressed as the mean \pm SD $(n=6)$.

Murine monocyte/macrophage cell line RAW264.7 was generously provided by the Department of Biological Sciences of Nanjing University. Cells were seeded in a 6-well plate $\left(1 \times 10^{6}\right.$ cells/well), maintained in a-MEM supplemented with $10 \%$ FBS, and pretreated with vehicle (DMSO) or with $5 \mu \mathrm{mol} / \mathrm{L}$ QOA-8a and stimulated with RANKL in a time-course experiment. The activating phosphorylation of ERK1/2, JNK, p38, p65, and IкBa was analyzed by Western blot. RAW264.7 cell cultures incubated in vehicle or with QOA-8a in the presence of RANKL for 1 or 2 days, proteins and mRNA expression of 
A

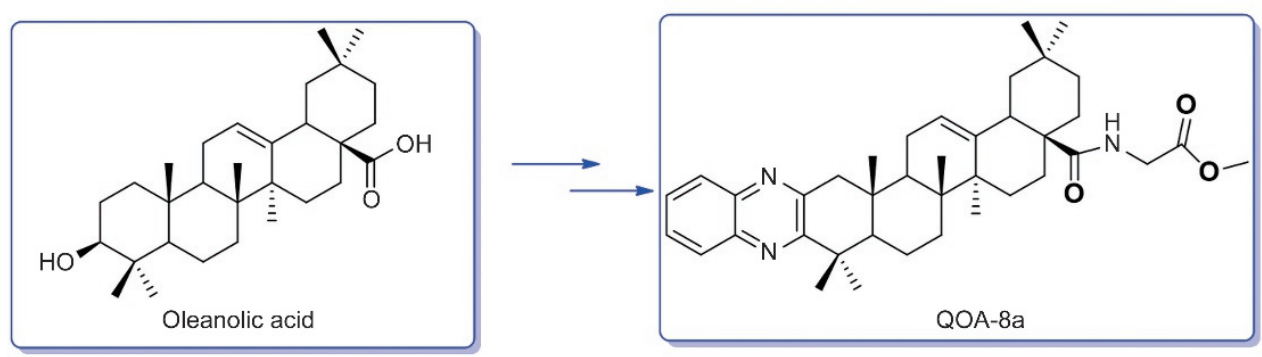

B

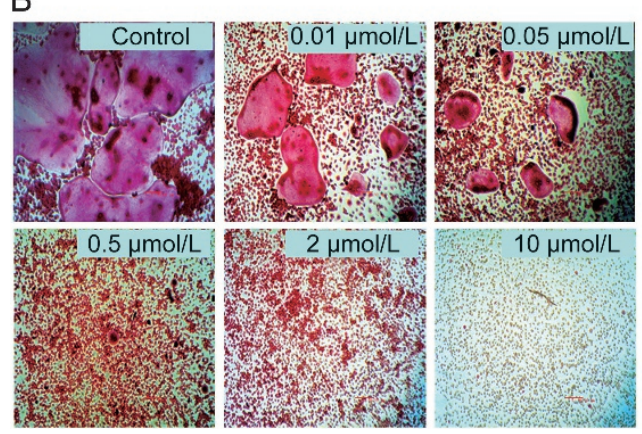

C

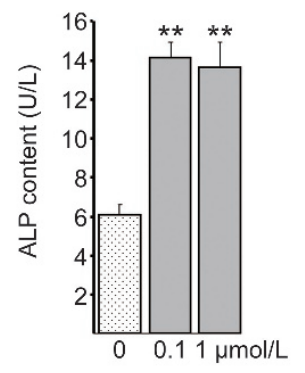

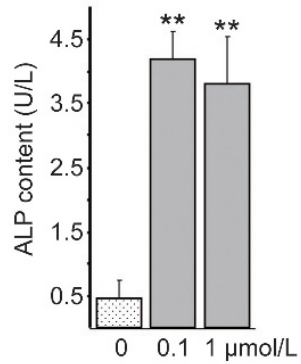

Figure 1. QOA-8a suppressed the formation of osteoclasts and stimulated differentiation of osteoblasts. (A) Chemical structure of QOA-8a. (B) Representative TRAP staining in cells; Primary bone marrow-derived macrophages (BMMs) incubated on vehicle (DMSO) or with Q0A-8a (0.01, 0.05, 0.5, 2, and $10 \mu \mathrm{mol} / \mathrm{L})$ in the presence of M-CSF $(30 \mathrm{ng} / \mathrm{mL})$ and RANKL $(50 \mathrm{ng} / \mathrm{mL})$ for $5 \mathrm{~d}$. Mean \pm SD. $n=6$. TRAP ${ }^{+}$osteoclast number at control group was 297 (×100). (C) Effects of QOA-8a on the ALP activity in mouse calvarial osteoblastic cell line MC3T3-E1. ALP in aliquots of supernatants (left) and cell lysates (right) were measured by an ALP activity kit, respectively. Mean \pm SD. $n=3 .{ }^{*} P<0.05,{ }^{* *} P<0.01$.

c-Fos and NFATc1 were analyzed by Western blot and realtime qPCR.

Human multinucleated osteoclast culture: Human peripheral blood mononuclear cells were separated from peripheral blood obtained from healthy volunteers by density gradient centrifugation with Ficoll-Paque Plus (Amersham Biosciences) using a protocol approved by Nanjing Drum Tower Hospital Affiliated with Medical School of Nanjing University. Informed consent was obtained from all subjects. Cells $\left(1 \times 10^{5}\right.$ cells/well) were plated in 96-well culture dishes in a-MEM supplemented with penicillin, streptomycin, 10\% FBS, 100 $\mathrm{ng} / \mathrm{mL}$ human RANKL and $50 \mathrm{ng} / \mathrm{mL}$ human M-CSF for 14 d. Cultures were refreshed twice weekly. After two weeks, the cells were stained with TRAP staining. Data are expressed as the mean $\pm \mathrm{SD}(n=3)$. Human peripheral blood mononuclear cells were seeded in a 6 -well plate $\left(1 \times 10^{6}\right.$ cells/well), pretreated with vehicle (DMSO) or with $5 \mu \mathrm{mol} / \mathrm{L}$ QOA-8a and stimulated with RANKL in a time-course experiment. The activating phosphorylation of ERK1/2, JNK, p38, p65, ІкBa was analyzed by Western blot.

\section{Osteoblast assay}

The mouse calvarial osteoblastic cell line MC3T3-E1 was purchased from the cell bank of type culture collection of the Chinese Academy of Sciences (Shanghai, China). Cells were seeded in a 6-well plate $\left(1 \times 10^{6}\right.$ cells/well $)$ and maintained in a-MEM supplemented with $10 \%$ FBS and antibiotics (penicillin $50 \mathrm{IU} / \mathrm{mL}$ and streptomycin $50 \mu \mathrm{g} / \mathrm{mL}$ ). MC3T3-E1 was treated with vehicle (DMSO) or QOA-8a in a time-course experiment. The activating phosphorylation of ERK1/2, JNK, p38 was analyzed by Western blot, and the expression of relevant genes was analyzed using real-time qPCR.

Isolation of mouse bone marrow mesenchymal stem cells: 5-week-old male ICR mice were used following protocols approved by the Institutional Animal Use and Care Committee of Nanjing University. Isolation of primary BMMSCs was performed according to the method described ${ }^{[26]}$. Briefly, the tibias and femurs were harvested, and the marrow was flushed by a syringe with a 26-g needle containing PBS. Cells were plated into a 6-well plate with a density of $1 \times 10^{7}$ cells per well in DMEM containing 10\% FBS. When primary cultures became nearly confluent, cells were harvested and cultured in a new $10-\mathrm{cm}$ petri dish. Once $70 \%-80 \%$ confluence was reached, cells were harvested for further experiments. For osteogenesis, DMEM was supplemented with $10 \mathrm{mmol} / \mathrm{L}$ $\beta$-glycerol phosphate from Sigma (St Louis, MO, USA), 50 $\mu \mathrm{g} / \mathrm{mL}$ ascorbate-2-phosphate from Sigma (St Louis, MO, USA) and $10^{-7} \mathrm{~mol} / \mathrm{L}$ dexamethasone from Sigma (St Louis, MO, USA). The medium was changed two times per week. After 7 d, cells were stained with Alizarin Red S from Sigma (St Louis, MO, USA). Alizarin Red staining was performed as follows: samples were fixed with $4 \%$ paraformaldehyde-PBS, washed with PBS three times and stained with $40 \mathrm{mmol} / \mathrm{L}$ Alizarin Red S ( $\mathrm{pH}$ 4.0) for $15 \mathrm{~min}$. Cells were rinsed for 15 min with PBS and then captured with a light microscope.

The human fetal osteoblast cell line hFOB1.19 was pur- 
chased from the cell bank of type culture collection of the Chinese Academy of Sciences (Shanghai, China). hFOB1.19 were seeded in a 96-well plate $\left(1 \times 10^{4}\right.$ cells/well $)$ and maintained in D/F12 supplemented with $10 \%$ FBS and antibiotics (penicillin $50 \mathrm{IU} / \mathrm{mL}$ and streptomycin $50 \mu \mathrm{g} / \mathrm{mL}$ ). The cells were pretreated with vehicle (DMSO) or with QOA-8a for $24 \mathrm{~h}$, and ALP, BMP-2, and OPG were then measured with corresponding kits. hFOB1.19 cells were seeded in a 6-well plate $\left(1 \times 10^{6}\right.$ cells/well), pretreated with vehicle (DMSO) or QOA-8a in a time-course experiment, and activating phosphorylation of ERK1/2, JNK, p38 was analyzed by Western blot.

QOA-8a was dissolved in DMSO followed by dilution with the appropriate medium to desired concentrations, and the DMSO final concentration was $0.1 \%$ in all the cultures. DMSO at $0.1 \%$ was added to normal, control groups and showed no effects on cells. All the cultures were kept in a $\mathrm{CO}_{2}$ incubator under moist conditions of $5 \% \mathrm{CO}_{2}$ in air at $37^{\circ} \mathrm{C}$.

\section{Conditioned media and sera analysis}

The levels of ALP in culture supernatants was directly quantified by an ALP kit from Jiancheng (Nanjing, China) according to the manufacturer's instructions. Cells in each group were lysed, the amount of total extracted protein was determined by a $\mathrm{BCA}^{\mathrm{TM}}$ protein assay kit (Pierce, Rochford, IL, USA), and the levels of BMP-2 and OPG were quantified by an ELISA kit from R\&D Systems (Minneapolis, USA) according to the manufacturer's instructions. Serum levels of N-MID-OT and CTX-I from rats in each group was determined using an ELISA kit from R\&D Systems (Minneapolis, USA) according to the manufacturer's instructions. The coefficient of variation (CV) of CTX-I for within-run assays was $6 \%$, and the range of measurement was $123.5-10000 \mathrm{pg} / \mathrm{mL}$. The CV of N-MID-OT for within-run assays was $5 \%$, and the range of measurement was $12-480 \mathrm{ng} / \mathrm{mL}$.

\section{Western blotting}

Cells were lysed in radioimmunoprecipitation assay (RIPA) buffer. The amount of total extracted protein was determined by a $\mathrm{BCA}^{\mathrm{TM}}$ protein assay kit (Pierce, Rochford, IL, USA). A quantity of $20-30 \mu \mathrm{g}$ of lysates was subjected to a $10 \%$ gradient SDS-polyacrylamide gel electrophoresis (SDS-PAGE) and transferred to a polyvinylidene difluoride membrane (Millipore, Bedford, MA, USA). Blots were probed with the primary antibody for the indicated proteins overnight at $4{ }^{\circ} \mathrm{C}$ and washed and incubated with the appropriate horseradish peroxidase (HRP)-conjugated secondary antibody for $1 \mathrm{~h}$ at room temperature. The blots were subsequently detected with an enhanced chemoluminescence kit according to the manufacturer's instructions (Cell Signaling Technology, MA, USA).

\section{Quantitative real-time PCR}

Total RNA was extracted using an RNeasy plus kit (QIAGEN), and cDNA was generated with an oligo (dT) primer and the Superscript II system (Invitrogen), followed by analysis using an iCycler PCR with SYBR Green PCR master mix (BioRad, CA, USA). Threshold cycle numbers were obtained using
BioRad CFX Manager software. The program for amplification was 1 cycle of $95^{\circ} \mathrm{C}$ for 2 min followed by 40 cycles of $95^{\circ} \mathrm{C}$ for $10 \mathrm{~s}, 60^{\circ} \mathrm{C}$ for $30 \mathrm{~s}$, and $95^{\circ} \mathrm{C}$ for $10 \mathrm{~s}$. The following primer sets were used: ALP (Forward: $5^{\prime}$-CGGGACTGGTACTCGGATAA-3'; Reverse: 5'-ATTCCACGTCGGTTCTGTTC-3'), BMP-2 (Forward: 5'-GCTCCACAAACGAGAAAAGC-3'; Reverse: 5'-AGCAAGGGGAAAAGGACACT-3'), collagen-type 1 (Forward: 5'-AGAGCATGACCGATGGATTC-3'; Reverse: 5'-CCTTCTTGAGGTTGCCAGTC-3'), RANKL (Forward: 5'-CCAAGATGGCTTCTATTACC-3'; Reverse: 5'-TCCCTCCTTTCATCAGGTTAT-3'), Runx2 (Forward: 5'-AGTAGCCAGGTTCAACGAT-3'; Reverse: 5'-GGAGGATTTGTGAAGACTGTT-3'), OPG (Forward: 5'-AGTCTGAGGAAGACCATGAG-3'; Reverse: 5'-AAACAGCCCAGTGACCATTC-3'), osteocalcin (Forward: 5'-CCTCAGTCCCCAGCCCAGATC-3'; Reverse: 5'-CAGGGCAGAGAGAGAGGACAG-3'), TRAP (Forward: 5'-TCTCCAAGCGCTGGAACTTC-3'; Reverse: 5'-CATTGGTCTGTGGGATCTTGAA-3'), cathepsin K (Forward: 5'-CACTGGATAATTAAAAACAGCTGGG-3'; Reverse: 5'-CCAGGTTGGCAATGCCAC-3'), c-Fos (Forward: 5'-ATGATGTTCTCGGGTTTCAACG-3'; Reverse: 5'-CAGTCTGCTGCATAGAAGGAACCG- ${ }^{\prime}$ ), and NFATc1 (Forward: 5'-GGGTCAGTGTGACCGAAGAT-3'; Reverse: 5'-GGAAGTCAGAAGTGGGTGGA-3').

\section{Animals}

Three-day-old ICR (Institute for Cancer Research) mice, 3-week-old male ICR mice, 5-week-old male mice and 8-weekold female Sprague-Dawley rats were purchased from the National Resource Center for Rodent Laboratory Animals. Animals were housed in a climate-controlled room, with a 12-h light/dark photoperiod. All the animals had free access to food and water.

All experimental protocols involving animals were approved by the Institutional Animal Care and Use Committee of Nanjing University. Animal welfare and all experimental protocols were performed strictly in accordance with the Guide for the Care and Use of Laboratory Animals. All efforts were made to minimize animals' suffering and to reduce the number of animals used.

\section{Bone histomorphometry}

Neonatal murine calvarias in organ culture: Calvarias from 3-day-old ICR mice were explanted, dissected free of adjacent connective tissue, placed in 12-well plates, incubated with QOA-8a, cultured at $37^{\circ} \mathrm{C}$ in a humidified atmosphere of $5 \% \mathrm{CO}_{2}$, and maintained in a-MEM supplemented with $10 \%$ FBS and antibiotics (penicillin $50 \mathrm{IU} / \mathrm{mL}$ and streptomycin 50 $\mu \mathrm{g} / \mathrm{mL}$ ). After $7 \mathrm{~d}$, the samples were stained with hematoxylin and eosin/Orange-G staining according to standard protocols. New bone area and osteoblast number were analyzed using a reflective optical microscope and the Metamorph 6.2 software $(\mathrm{M} \times 50$, Olympus, Guangzhou, China). Murine calvarias in vivo: Male ICR mice, 3 weeks of age, were injected two times per day for $10 \mathrm{~d}$ over the right side of the calvaria with either 
vehicle or QOA-8a. After the experiment, the calvarias were removed and stained with hematoxylin and eosin/Orange-G staining. New bone area and osteoblast number were analyzed using a reflective optical microscope and the Image-Pro Plus software. Treatment effect on OVX: 8-week-old female rats were subjected to either bilateral ovariectomy (OVX) or a sham operation. After the surgical operation, OVX-rats were left untreated for 8 weeks and then treated orally with vehicle or the indicated dose of QOA-8a and AS for 8 weeks. Protective effect on OVX: Three days after the surgical operation, the 8-week-old female rats were administered either vehicle, a relatively dose of AS or increasing amounts of QOA-8a for 16 weeks. The trabecular bone architecture of the proximal femur and vertebra were analyzed using microcomputed tomography (SSRF, Shanghai Synchrotron Radiation Facility). The total BMD, vertebral BMD, right proximal femoral BMD and right proximal femoral BMD was measured by dualenergy X-ray absorptiometry (DXA) (Lunar Co, Madison, WI, USA) under pentobarbital anesthesia (the reproducibility of the DXA procedure on the rat femur had a coefficient of variation of $1.21 \%$ of six measurements). The left proximal femurs were scanned with a microcomputed tomography $(\mu \mathrm{CT})$ system (Line BL13W1, Shanghai Synchrotron Radiation Facility, Shanghai, China).

\section{Statistical analyses}

Data are presented as the mean $\pm \mathrm{SD}$ of the indicated number of experiments. Data were analyzed by one-way ANOVA followed by Dunnett's test or Student's $t$-test if appropriate. Probability values of 0.05 or less were considered statistically significant. All statistical analyses were performed with SPSS 13.0 (SPSS Inc, Chicago, IL, USA).

\section{Results}

\section{QOA-8a inhibits RANKL-induced osteoclast formation}

We previously demonstrated that QOA-8a significantly inhibited the differentiation, formation and bone resorptive activity of osteoclasts without cytotoxicity ${ }^{[24]}$. To further confirm the effects of QOA-8a on osteoclast activity, we assayed the half inhibitory concentration $\left(\mathrm{IC}_{50}\right)$ of QOA-8a. Primary bone marrow-derived macrophages (BMMs) were treated with various concentrations of QOA-8a in the presence of RANKL and M-CSF, two essential factors that regulate the development of osteoclasts. Formation of multinucleated osteoclasts was determined by tartrate-resistant acidic phosphatase (TRAP) staining. The result showed that QOA-8a exhibited an $\mathrm{IC}_{50}$ value of $0.098 \mu \mathrm{mol} / \mathrm{L}$ (Figure 1B). Next, to determine the stage of the main effects of QOA-8a in osteoclast formation , QOA-8a $(2 \mu \mathrm{mol} / \mathrm{L})$ was added to the medium on each day from the first to the fifth day, respectively, and then, on each of the following days, QOA-8a was washed out and normal differentiated conditions were allowed to recover (Figure 2A). When QOA-8a was added on the fourth day, the inhibitory effect on osteoclast formation was the greatest $(94.4 \%$, Figure 2B). The results suggested that the potent inhibitory effect of QOA-8a on osteoclast formation was mainly exerted during the highly differentiating period.

QOA-8a stimulates new bone formation in murine calvarial bones in vitro and in vivo

Our research clearly demonstrated that QOA-8a could stimulate osteoblast differentiation (Figure 1C). To examine this effect, we investigated the anabolic actions of QOA-8a on neonatal murine calvarial bones in an organ culture. Bones were isolated from 3-day-old ICR mice (Institute for Cancer Research $)^{[27,28]}$ and placed in the conditioned media after they were dissected free of adjacent connective tissue. After incubation with QOA-8a for 7 days, a marked increase in new bone formation was observed in a dose-dependent manner compared with the control (Figure 3A and 3B). Moreover, the marked stimulatory effect of QOA-8a on bone cells has also been found in conjunction with enhanced ALP activity (Figure 1C, Figure 3C). We therefore concluded that QOA-8a possessed dual effects both on osteoclasts and osteoblasts.

To further confirm the bone anabolic effect, we injected QOA-8a into the subcutaneous tissue overlying the murine calvaria in vivo with a careful experimental operation ${ }^{[27]}$. Three-week-old male ICR mice were injected twice daily over
A

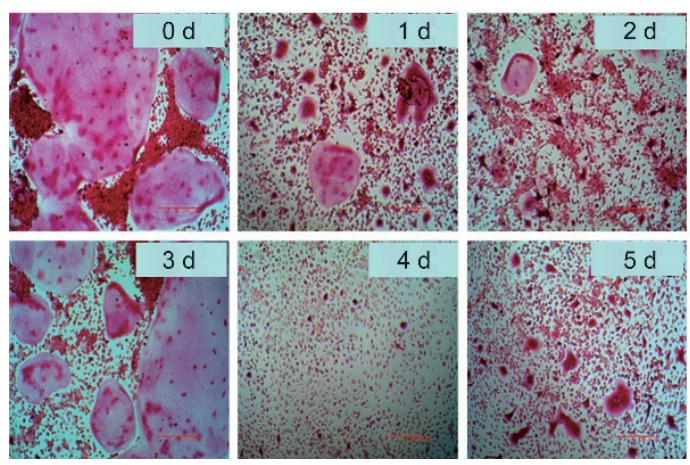

$\mathrm{B}$

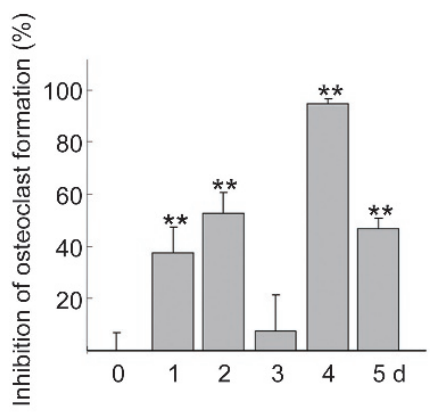

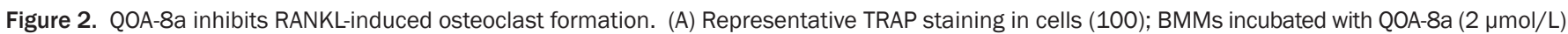

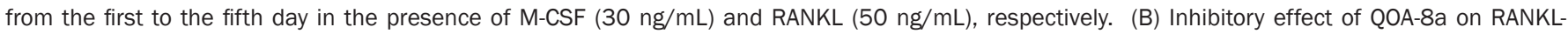
induced osteoclast formation at first to fifth day. Mean \pm SD. $n=6$. ${ }^{* *} P<0.01$ vs d 0 . 
A

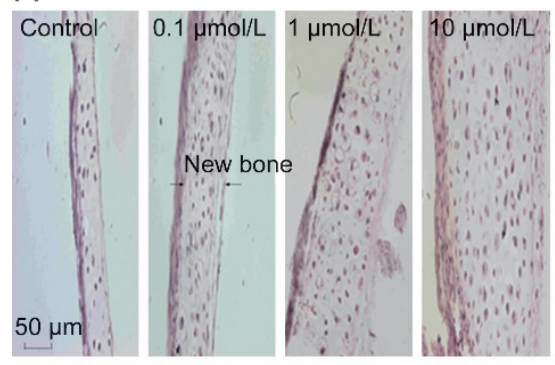

B

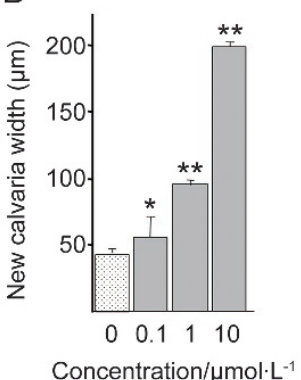

C

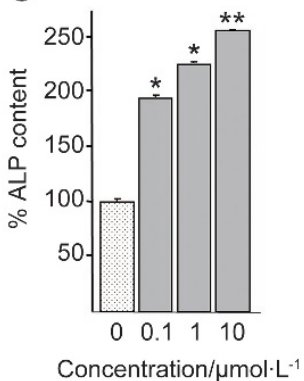

D

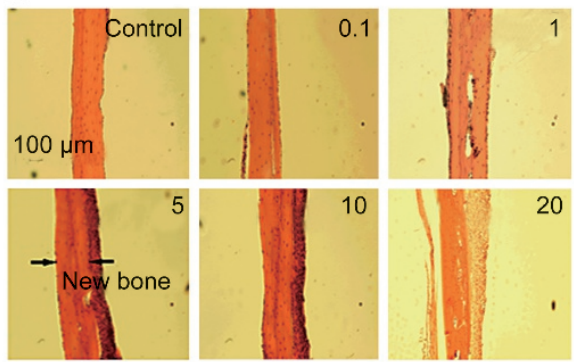

$\mathrm{F}$

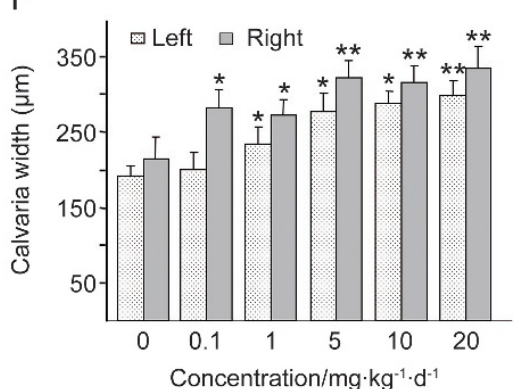

E

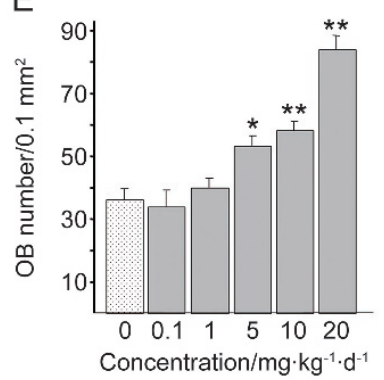

G

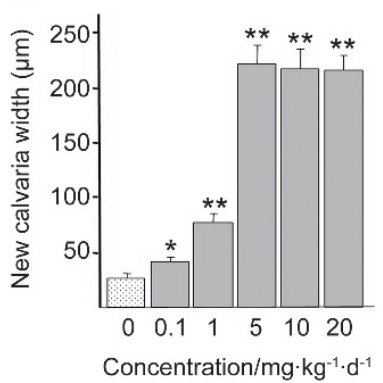

Figure 3. QOA-8a stimulates new bone formation on murine calvaria in vitro and in vivo. (A) Effects of QOA-8a added to cultures of explanted murine calvaria for 7 days. (B) Quantitative effects of QOA-8a on the width of new calvarial bones. (C) The culture medium was subjected to ALP activity. Mean \pm SD. $n=6$. ${ }^{*} P<0.05,{ }^{* * *} P<0.01$ vs d 0 . (D) Effect of QOA-8a $0.1-10 \mathrm{mg} \cdot \mathrm{kg}^{-1} \cdot \mathrm{d}^{-1}$ on new bone formation after local subcutaneous injection over the murine calvaria. (E) Osteoblasts numbers (per $0.1 \mathrm{~mm}^{2}$ bone) of the right calvaria. (F) The width of the right and left calvarial bone, after local subcutaneous injection over the right side of the calvaria in mice, with either vehicle or QOA-8a for $10 \mathrm{~d}$. (G) Increased new bone width of the right calvaria (the injected side). Mean \pm SD. $n=5 .{ }^{*} P<0.05,{ }^{* *} P<0.01$ vs d 0 .

the right side of the calvaria with either vehicle or QOA-8a. After being injected subcutaneously for $10 \mathrm{~d}$, the calvaria were removed for histomorphometric analysis. As we observed, increased QOA-8a levels were accompanied by increased osteoblast activity both in the right and left side of the calvaria (Figure 3D), which is an almost 7-fold increase in new calvarial width (Figure 3F-G), and a 2-fold increase in osteoblast number (Figure 3E) occurred compared with the control group. The results demonstrated that QOA-8a treatment possessed a marked bone anabolic effect in a dose-dependent manner in vivo.

\section{Treatment effect of QOA-8a on OVX-induced bone loss}

To confirm the treatment effect of QOA-8a on OVX induced bone loss, we ovariectomized rats at 8 weeks of age and left them untreated for 8 weeks so that they developed severe osteopenia. Then, sham-operated or OVX rats were treated for 8 more weeks with either vehicle or increasing amounts of QOA-8a $\left(0.1,1\right.$ or $10 \mathrm{mg} \cdot \mathrm{kg}^{-1} \cdot \mathrm{d}^{-1}$, orally), and alendronate sodium (AS, $1 \mathrm{mg} \cdot \mathrm{kg}^{-1} \cdot \mathrm{d}^{-1}$, orally) acted as a positive control. The total bone mineral density (BMD) and the BMD of the right femur, vertebra and proximal right femur of each of the 8 rats from the sham and OVX groups were measured with dual-energy X-ray absorptiometry (DXA). After 8 weeks, the $\mathrm{BMD}$ of the sham and randomly selected $8 \mathrm{OVX}$ rats were measured with DXA.

As shown in Figure 4A, significant reductions in total BMD and BMD in vertebra and proximal right femur were observed.
The body weight of OVX rats increased by approximately $15 \%$ compared with that of sham-operated rats $(P<0.05)$ (Figure 4B). The uterine weight of OVX rats substantially decreased by $78.5 \%$ compared with that of sham-operated rats $(P<0.01)$, which indicated that the rats were estrogen deficient and confirmed the success of the ovariectomy procedure (Figure 4C). The results indicated an improvement to the postmenopausal osteoporotic state. The eight-week treatment of QOA-8a at all dose levels did not elicit any hormonelike side effects, and there was no degeneration or necrosis of endometrial glands and smooth muscle cells and no infiltration of inflammatory cells (Figure 4D).

Because the proximal femurs in humans and rats share many histoanatomic similarities, we first used $\mu \mathrm{CT}$ to investigate whether QOA-8a has a role in increasing femoral trabecular microarchitecture. Right proximal femurs were used for bone histomorphometry; ovariectomy decreased femoral trabecular bone volume (Figure 4E), trabecular thickness (Figure $4 \mathrm{~F}$ ), and increased trabecular separation (Figure 4G). As expected, daily administration with QOA-8a in the 1 and 10 $\mathrm{mg} \cdot \mathrm{kg}^{-1} \cdot \mathrm{d}^{-1}$ treatments for 8 weeks reversed the effects of OVX significantly and prevented further deterioration of the bone structure (Figure $4 \mathrm{H}$ ).

\section{Protective effect of QOA-8a on OVX-induced bone loss}

We further evaluated the preventive effect of QOA-8a on OVX-induced bone loss as the treatment was started 

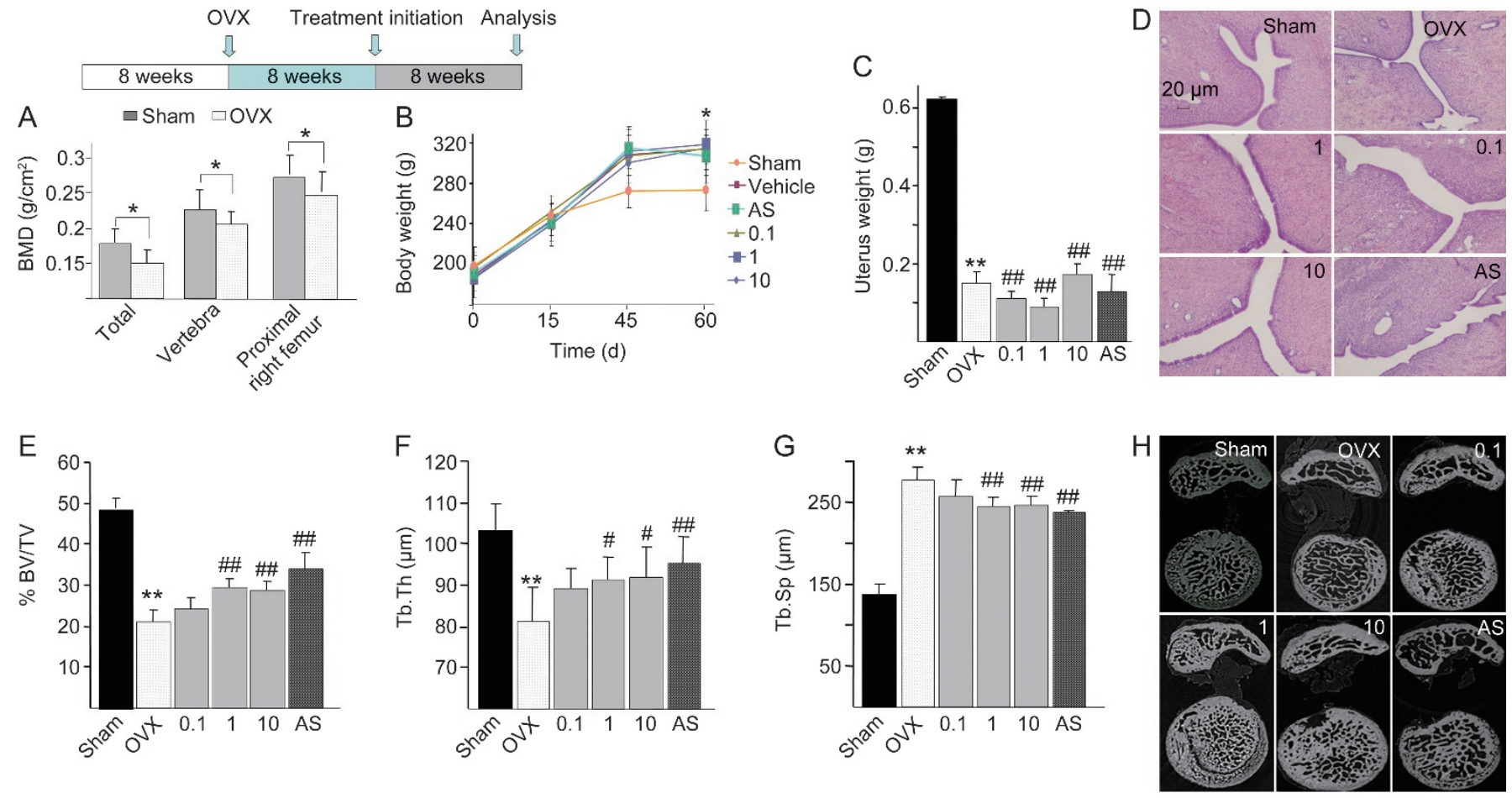

Figure 4. QOA-8a leads to an increase in trabecular microarchitecture. OVX rats were left untreated for 8 weeks, then treated orally with vehicle or the indicated dose of QOA-8a and AS ( $1 \mathrm{mg} \cdot \mathrm{kg}^{-1} \cdot \mathrm{d}^{-1}$, orally) for 8 weeks. Mean \pm SD. $n=8$. ${ }^{*} P<0.05,{ }^{* *} P<0.01$ vs sham group. ${ }^{\#} P<0.05$, ${ }^{\# \#} P<0.01 \mathrm{vs} 0 \mathrm{VX}$ group. (A) The change of BMD between OVX groups and sham group, measured by DXA after surgical procedure, before treated with QOA-8a. (B) Body weight of OVX groups and sham group after the surgical procedure. (C) Uterine wet weight of OVX groups and sham group after euthanized. (D) The H\&E-stained uterine sections ( $\times 100$ ), from the different OVX-QOA-8a groups, OVX-AS group and OVX-vehicle groups. (E-G) Bone morphometric analysis of right proximal femurs from sham and OVX rats. BV/TV, bone volume/total volume (E); Tb.Th, trabecular bone thickness (F); Tb.Sp, trabecular bone separation $(\mathrm{G})$. (H) Microstructural analysis of trabecular areas of right proximal femurs measured by $\mu \mathrm{CT}$.

immediately after ovariectomy. The female rats $(8$ weeks old) were sham-operated or ovariectomized and then treated with either vehicle, AS $\left(1 \mathrm{mg} \cdot \mathrm{kg}^{-1} \cdot \mathrm{d}^{-1}\right.$, orally) or increasing amounts of QOA-8a (1 or $5 \mathrm{mg} \cdot \mathrm{kg}^{-1} \cdot \mathrm{d}^{-1}$, orally) for 16 weeks. The efficiency of OVX was assessed by the significant weight gain (approximately 21\%) compared with that of sham rats (Figure 5A), and the uterine weight of OVX rats also showed a marked reduction (Figure 5B) due to uterine atrophy. After the experiment, we first measured cortical and cancellous bone mineral densities of the left proximal femurs with peripheral quantitative computed tomography (pQCT). As we observed, the bone mineral densities of the proximal femur were significantly decreased in the OVX groups, and QOA-8a treatment displayed a significant effect in restoring the deterioration of bone mass in OVX rats (Figure 5C). The dosage of $1 \mathrm{mg} \cdot \mathrm{kg}^{-1} \cdot \mathrm{d}^{-1}$ showed the most significant reversal of deleterious effects, which was higher than that in shamoperated rats. Notably, QOA-8a increased both cortical and cancellous bone mineral densities in ovariectomized rats compared to vehicle-treated controls (Figure 5D, 5E).

Bone turnover can be estimated by markers of bone formation (N-MID Osteocalcin, N-MID-OT) and bone resorption (cross-linked C-telopeptide of type I collagen, CTX-I). These markers have a rapid therapeutic response to treatment, especially compared to BMD measurement, that can be used to predict the rate of bone loss in postmenopausal women and can be used to assess the risk of fractures ${ }^{[29]}$. Next, we examined the role of QOA-8a in bone metabolism in vivo. We first investigated the serum levels of the bone resorption marker, CTX-1. Ovariectomy led to a substantially increased level of the bone resorption marker and high rates of bone turnover. This increase was reversed to equivalent degrees by both AS and all doses of QOA-8a (Figure 5F). Due to this high bone turnover model, the serum levels of the osteoblast marker, N-MID-OT, were also elevated significantly in ovariectomized rats. Treatment with QOA-8a slightly increased the serum levels of N-MID-OT, whereas AS had no effects on this marker (Figure 5G). Analysis of these bone turnover markers demonstrated the anabolic and the antiresorptive effects of QOA-8a, which showed a dual role in promoting osteoblast activity and inhibiting osteoclast activity.

We further investigated two microarchitectural parameters, endosteal and periosteal circumference, at the left femoral shaft. As shown in Figure 5, both endosteal and periosteal circumference were larger, as expected, which was caused by increasing bone remodeling after ovariectomy. Alendronate sodium treatments decreased endosteal circumference but had little effect on periosteal circumference (Figure $5 \mathrm{H}$ ), indicating 
A

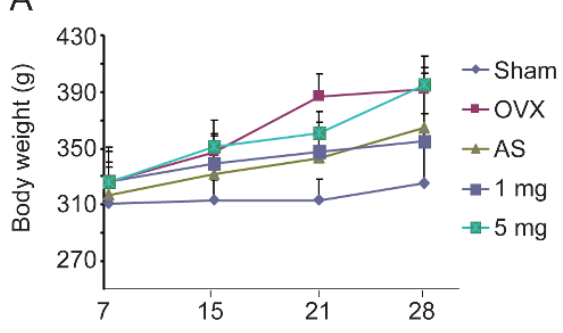

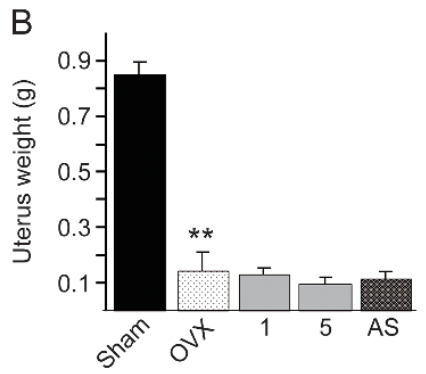
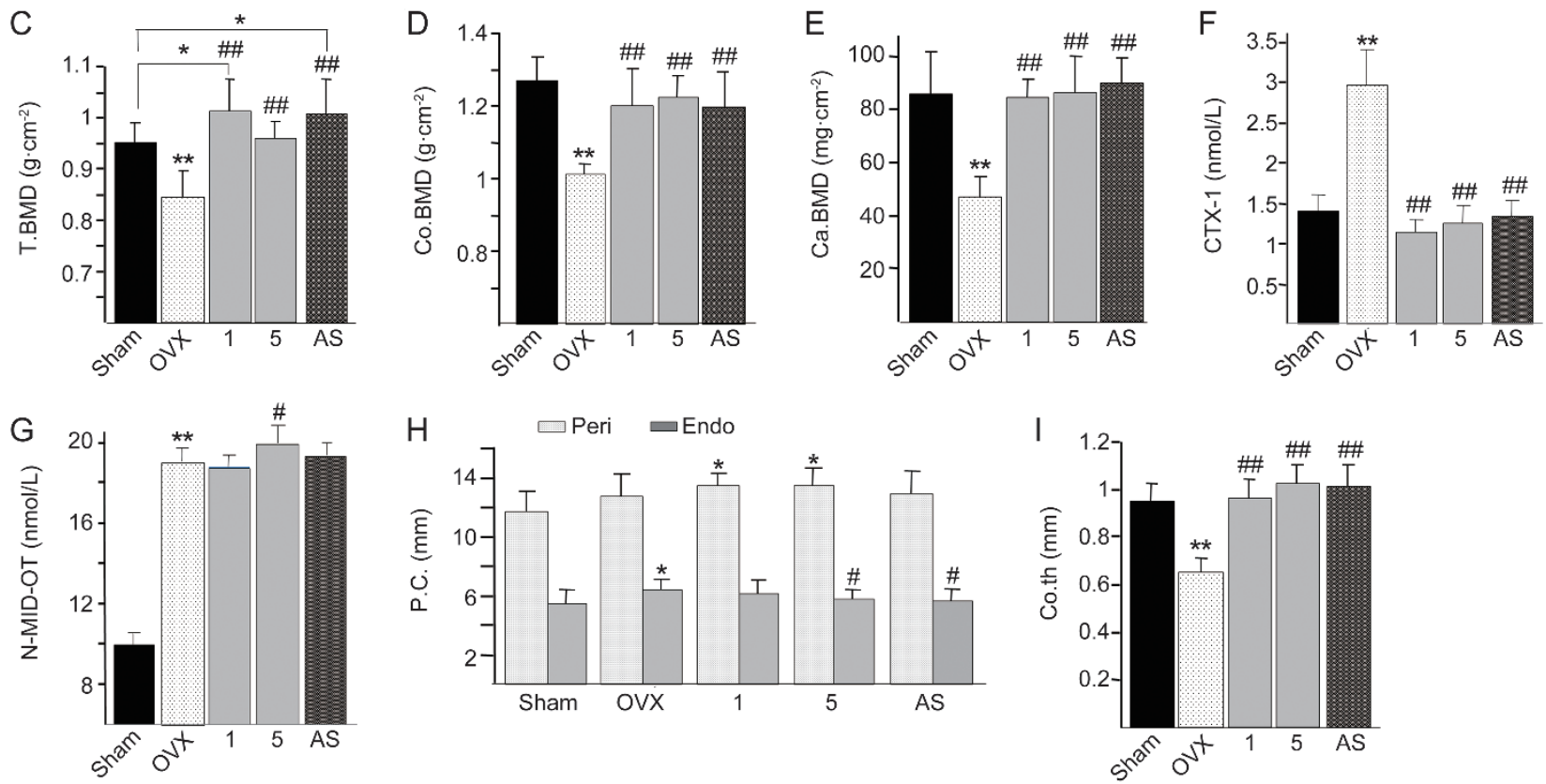

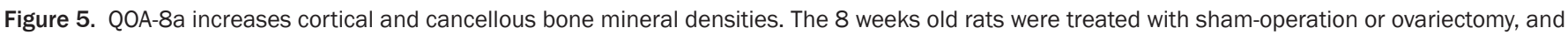
administered with either vehicle, a relatively dose of AS (1 mg. $\mathrm{kg}^{-1} \cdot \mathrm{d}^{-1}$, orally) or increasing amounts of QOA-8a (1 or $5 \mathrm{mg} \cdot \mathrm{kg}^{-1} \cdot \mathrm{d}^{-1}$, orally). (A) Body weight of OVX groups and sham group after the surgical procedure. (B) Uterine wet weight of OVX groups and sham group after euthanized. (C) The total bone mineral density (T.BMD) of right proximal femurs. (D) Cortical bone mineral densities (Co.BMD) in the right proximal femurs. (E) Cancellous bone mineral densities (Ca.BMD) in the right proximal femurs. ELISA assay for CTX-1 (F), N-MID-OT (G). (H) The endosteal and periosteal circumference at the left femoral shaft. (I) The cortical thickness at the left femoral shaft. Mean \pm SD. $n=10$. ${ }^{*} P<0.05,{ }^{* *} P<0.01$ vs sham group. ${ }^{\#} P<0.05,{ }^{\# \#} P<0.01$ vs 0 VX group.

the antiresorptive properties of the bisphosphonates. QOA-8a not only decreased endosteal circumference but also increased periosteal circumference, and the dosage of $5 \mathrm{mg} \cdot \mathrm{kg}^{-1} \cdot \mathrm{d}^{-1}$ showed the most significant effects, which indicated that QOA-8a could decrease endosteal bone resorption and increase periosteal bone formation (Figure $5 \mathrm{H}$ ). We also measured cortical thickness at the left femoral shaft, which was reduced in OVX rats because of the high endocortical resorption. All doses of QOA-8a reversed the decrease in cortical thickness to levels similar to $\left(1 \mathrm{mg} \cdot \mathrm{kg}^{-1} \cdot \mathrm{d}^{-1}\right)$ or higher than $\left(5 \mathrm{mg} \cdot \mathrm{kg}^{-1} \cdot \mathrm{d}^{-1}\right)$ those seen in sham-operated rats (Figure 5I).

\section{Signaling involved in inhibiting osteoclast formation by QOA-8a}

To gain insight into the preliminary mechanisms underlying the effect of QOA-8a on inhibiting osteoclast differentiation, we explored the activation status of key signals in signaling pathways involved in the regulation of this osteoclastogenesis. Upon stimulation by RANKL, QOA-8a induced a remarkable inhibition of ERK1/2 phosphorylation in the RAW264.7 cell line but slightly modulated p38-MAPK and JNK activation (Figure 6A). Because ERK1/2 and JNK activated c-Fos ${ }^{[30]}$ and NFATc $1^{[31]}$, respectively, we investigated whether QOA-8a affected the expression of the two transcription factors. The results demonstrated that QOA-8a significantly inhibited c-Fos mRNA levels (Figure 6C) and protein expression (Figure 6B) and only had a suppressive effect on NFATc1 protein expression but little effect on mRNA levels. In our experimental settings, QOA-8a inhibited the NF-кB-p65 signaling but failed to affect IKBa phosphorylation (Figure 6A).

Osteogenic effects and signaling involved in stimulating osteoblast differentiation by QOA-8a

We examined the biological effect of OOA-8a in promoting 

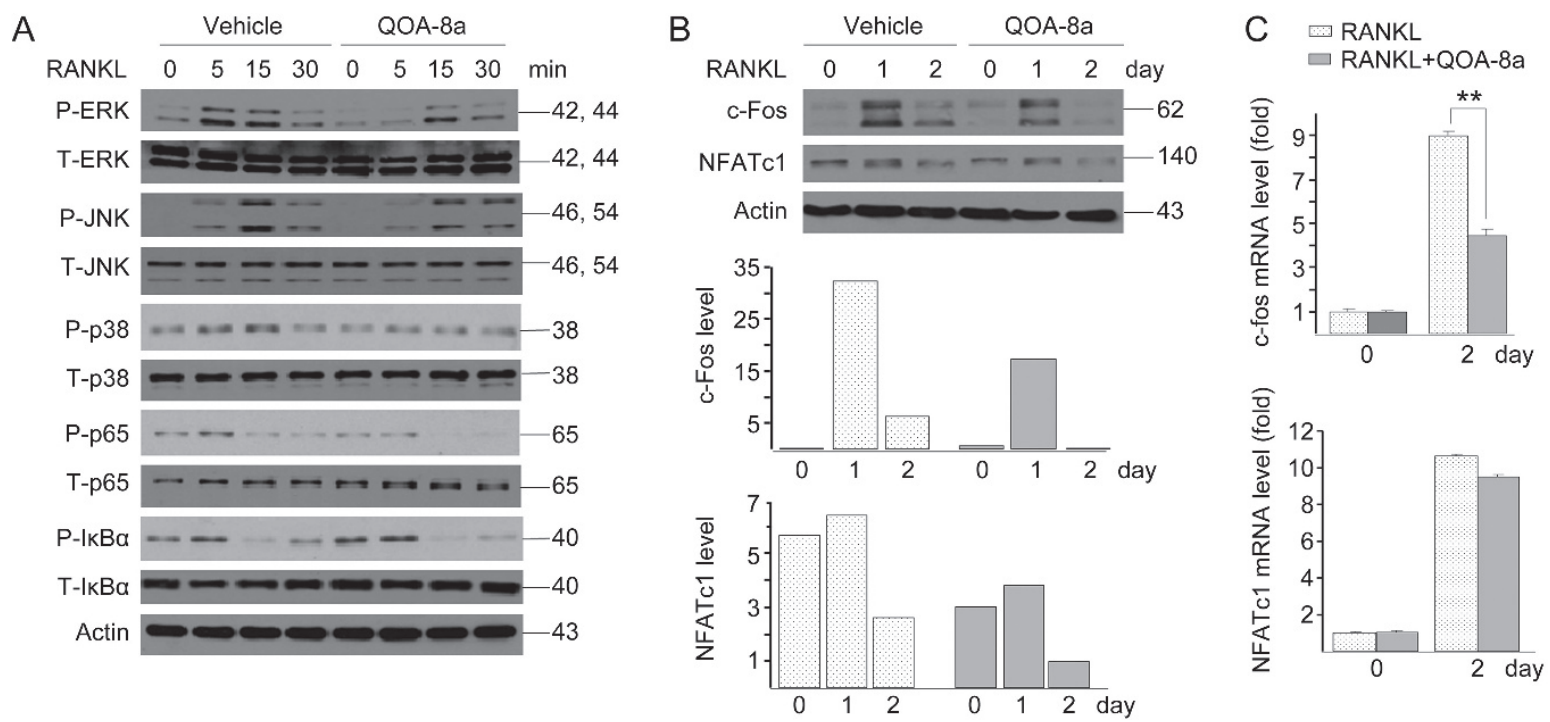

Figure 6. Signaling involved in inhibiting osteoclast formation induced by QOA-8a. (A) Osteoclast precursors (RAW264.7 cells) were pretreated with vehicle (DMSO) or with 5 mol/L QOA-8a and stimulated with RANKL in a time-course experiment, and Western blot analysis of ERK1/2, JNK, p38, p65, IKB $\alpha$ activating phosphorylation. (B) RAW264.7 cell cultures incubated on vehicle or with QOA-8a in the presence of RANKL for 1 or $2 \mathrm{~d}$. Western blot analysis of c-Fos and NFATC1 proteins, normalized versus actin. (C) Real-time qPCR of c-Fos and NFATc1 expression, normalized with actin expression. Mean \pm SD. $n=3$. ${ }^{* *} P<0.01$.

osteogenic differentiation by Alizarin Red staining and measuring ALP in osteoblasts differentiated from primary mouse bone marrow mesenchymal stem cells (BMMSCs) ${ }^{[26]}$. QOA-8a $(0.2$ or $2 \mu \mathrm{mol} / \mathrm{L})$ was added to the osteogenic medium at the initiation of osteoblast differentiation. Accordingly, as shown in Figure 7A, when the cells were induced to differentiate into different lineages, QOA-8a showed enhanced positive staining for alizarin red, and QOA-8a also significantly and dosedependently increased ALP activity in osteoblasts derived from primary BMMSCs. AS showed no osteogenic effects during osteoblast differentiation (Figure 7B).

Next, we examined the signaling involved in stimulating osteoblast differentiation by QOA-8a. Interestingly, QOA-8a significantly increased phosphorylation of JNK and ERK1/2 (Figure 7C), which were down-regulated by QOA-8a in osteoclast precursors upon exposure to RANKL, indicating that JNK and ERK1/2 signaling seemed to play an important dual role in how QOA-8a inhibited osteoclast differentiation and stimulated osteoblast differentiation. We further examined whether QOA-8a led to the activation of STAT3. Indeed, we observed that QOA-8a up-regulated STAT3 phosphorylation in osteoblasts (Figure 7D). These results suggested that QOA8a stimulates osteoblast differentiation through JNK, ERK/ STAT signaling.

To further demonstrate the effects of QOA-8a on promoting osteoblast function, we evaluated mRNA expression in the bone of the osteoblast markers ALP, Osteocalcin (OC), runt-related transcription factor 2 (Runx2), bone morphogenetic protein 2 (BMP-2), collagen-type 1 (Coll-1) and osteoprogesterin (OPG) (Figure 7D-L). The differentiation of the preosteoblast into a mature, bone-forming osteoblast phenotype requires the expression of Runx2 (Figure 7G); the mature osteoblast expresses the matrix proteins Coll-1(Figure 7I), OC (Figure 7F) and a key enzyme in the mineralization process, ALP (Figure 7E ${ }^{[32]}$. The up-regulated markers further indicated an increase in osteoblast activity by QOA-8a. Our findings also showed that the OPG expression level was significantly up-regulated (Figure 7J), and the RANKL level was down-regulated (Figure $7 \mathrm{~K}$ ) in the mouse calvarial osteoblastic cells, suggesting that QOA-8a impacted mouse osteoblasts to control osteoclast development and resorption through RANKL and OPG output (Figure 7L).

\section{Dual effects of QOA-8a both on human-sourced osteoclasts and osteoblasts}

To provide evidence that the dual role is not specific to the animal model, we further tested whether QOA-8a treatment affected both human-sourced osteoclasts and osteoblasts. We differentiated human peripheral blood mononuclear cells into osteoclasts in the presence of M-CSF $(50 \mathrm{ng} / \mathrm{mL})$ and RANKL $(100 \mathrm{ng} / \mathrm{mL})$. The formation of multinucleated osteoclasts was determined by TRAP staining. Treatment with QOA-8a resulted in a significantly reduced number of TRAP-positive osteoclasts and a reduced average number of nuclei per TRAPpositive cell in a concentration-dependent manner (Figure 8A-B). Moreover, at concentrations up to $50 \mu \mathrm{mol} / \mathrm{L}$, QOA8a did not show any cytotoxicity on the osteoclast precursors (Figure 8C).

To verify whether QOA-8a affected osteoblast differentiation, we treated human fetal osteoblast cells (hFOB1.19) with different concentrations of QOA-8a, and the data displayed that QOA-8a exhibited considerable ALP stimulatory activity in a concentration-dependent manner compared with that of the control value (Figure $8 \mathrm{E}$ and $8 \mathrm{~F}$ ). We also examined the pro- 
A
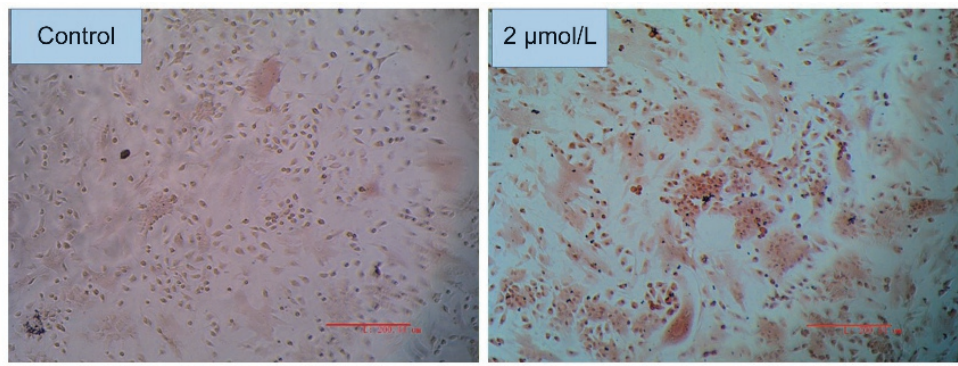

C
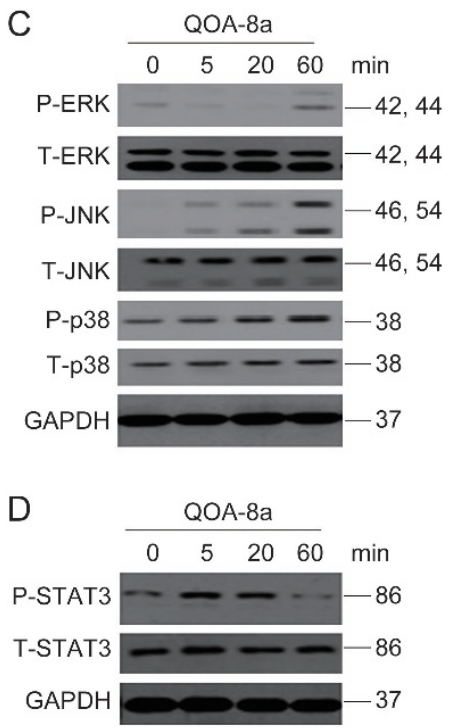

E
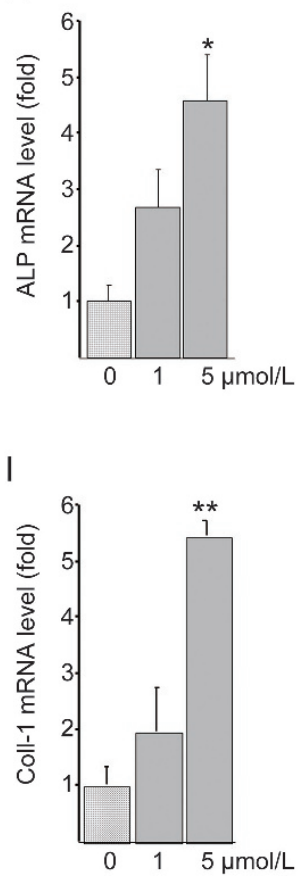

F

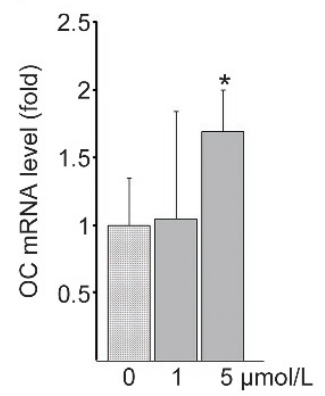

J

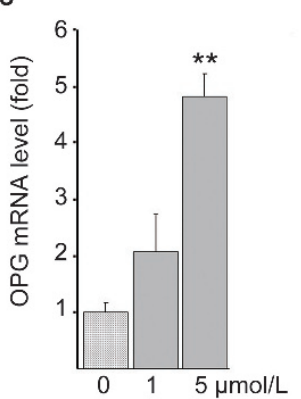

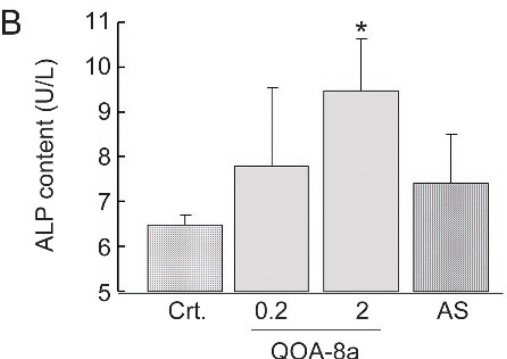

G

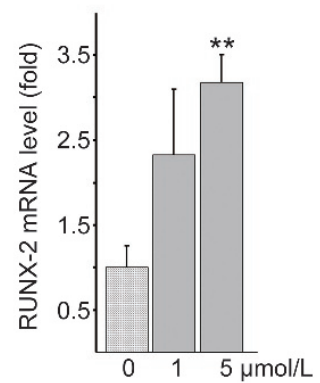

$\mathrm{H}$

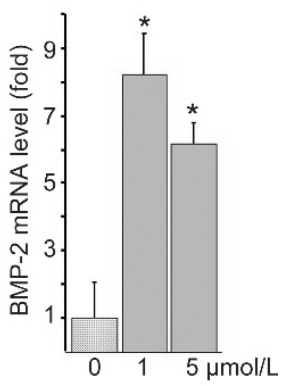

L

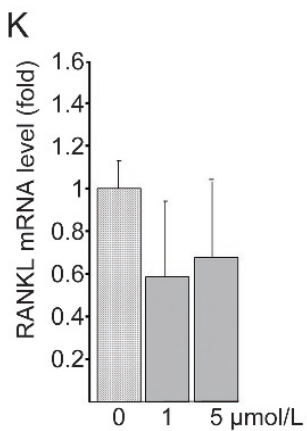

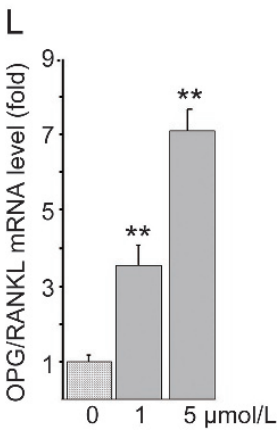

Figure 7. Osteogenic effects and signaling involved in stimulating osteoblast differentiation by QOA-8a. (A) The Alizarin Red staining on primary BMMSCs incubated with vehicle (DMSO) or with QOA-8a $(2 \mu \mathrm{mol} / \mathrm{L})$ in the presence of $\beta$-glycerol phosphate $(10 \mathrm{mmol} / \mathrm{L})$, ascorbate-2-phosphate $(50$ $\mu \mathrm{g} / \mathrm{mL})$ and dexamethasone $\left(10^{-7} \mathrm{~mol} / \mathrm{L}\right)$ for $7 \mathrm{~d}$. (B) Effects of QOA-8a on the ALP activity in osteoblast differentiated from primary BMMSCs for $7 \mathrm{~d}$. ALP in aliquots of supernatants from vehicle (DMSO), QOA-8a $(0.2,2 \mu \mathrm{mol} / \mathrm{L})$ or AS $(2 \mu \mathrm{mol} / \mathrm{L})$ was measured by an ALP activity kit. Mean \pm SD. $n=3$. ${ }^{*} P<0.05$. For signaling experiment, mouse calvarial osteoblastic cell line MC3T3-E1 was treated with $5 \mu \mathrm{mol} / \mathrm{L}$ QOA-8a in a time-course experiment, and Western blot analysis of ERK1/2, JNK, p38 activating phosphorylation (C), and STAT3 activating phosphorylation (D). (E-K) Osteoblast differentiation marker expression assessed by real-time qPCR in MC3T3-E1 cells treated with different doses of QOA-8a for $12 \mathrm{~h}$, normalized versus actin. Mean \pm SD. $n=3$. ${ }^{*} P<0.05,{ }^{* *} P<0.01$. (L) The ratio of OPG and RANKL mRNA expression.

tein expression of BMP-2 and OPG (Figure 8G, 8H) in human fetal osteoblast cells using an ELISA assay. Most of the markers were up-regulated by QOA-8a. From Figure 8, we can see that BMP-2 level was significantly expressed by QOA-8a in human fetal osteoblast cells (Figure 8G).

Next, we asked whether the signaling pathway described above is involved in this human osteoclastogenesis and osteoblastogenesis. We found that, similar to previous results, ERK1/2 and NF-kB-p65 were down-regulated by QOA-8a in human osteoclast precursors upon exposure to RANKL (Figure 8D), whereas ERK1/2 and p38-MAPK, but not JNK , were up-regulated by QOA-8a in human fetal osteoblast cells (Figure 8I). The data demonstrated that QOA-8a had an apparent effect on osteoblast development and osteoblast function.

\section{Discussion}

Our findings showed a novel small-molecule, QOA-8a, with dual-opposite roles in building up bones. We had already confirmed that QOA-8a inhibited RANKL-induced osteoclast formation and bone resorptive activity of mature osteoclasts without cytotoxicity ${ }^{[2]}$. In an attempt to demonstratethe anabolic actions, we added QOA-8a to neonatal murine calvarial bones in organ culture and injected QOA-8a into the subcutaneous tissue overlying the murine calvaria in mice. The results showed that QOA-8a treatment led to a marked bone anabolic effect in a dose-dependent manner.

Because osteoporosis occurs most frequently in 
A

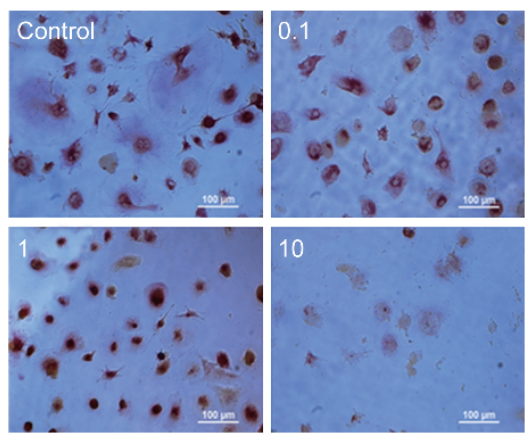

B

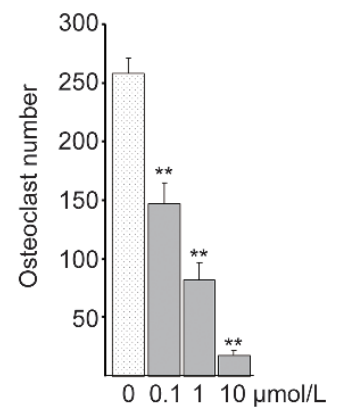

C

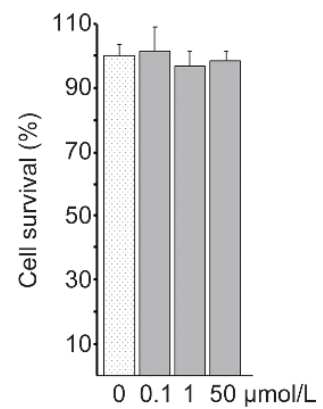

$D_{\text {RANKL }} \frac{\text { Vehicle }}{0 \quad 51530} \frac{\text { QOA-8a }}{051530}$ min

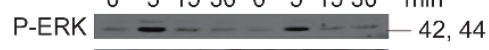

T-ERK 42,44

P-JNK - -ニ-ーーニ- $-46,54$

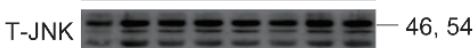

P-p38 -38

T-p38 - - - - - 38

P-p65 - - - - -65

T-p65 - - - - - - 65

P-ІкBa -40

Т-ІкBa $------2-40$

GAPDH -37
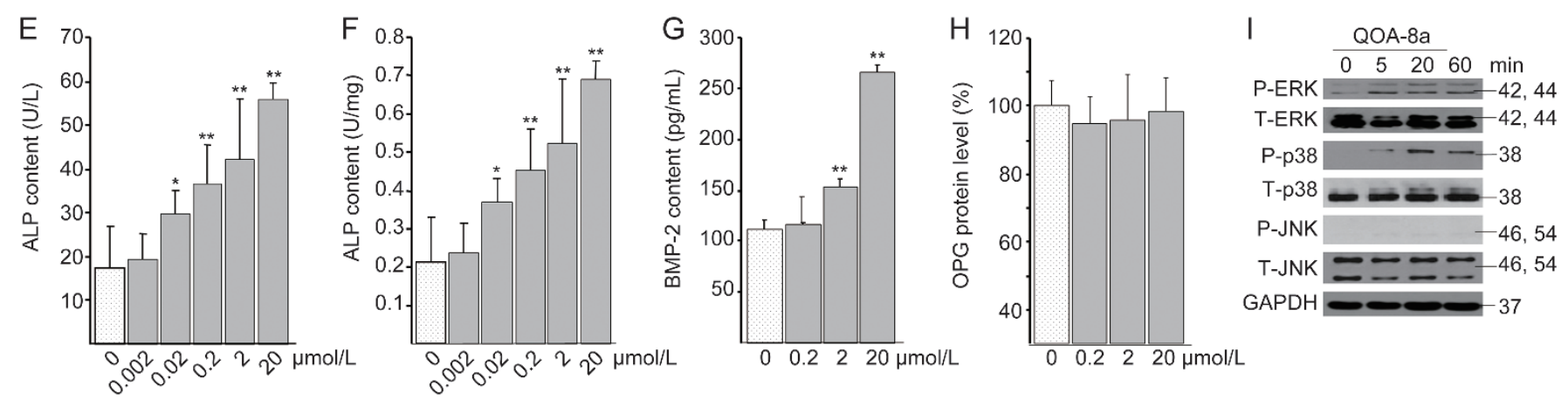

Figure 8. Dual effects of QOA-8a both on human-sourced osteoclasts and osteoblasts. (A) Representative TRAP staining in cells ( $\times 100)$; human peripheral blood mononuclear cells (PBMCs) incubated on vehicle (DMSO) or with QOA-8a 0.1-10 $\mu \mathrm{mol} / \mathrm{L}$ in the presence of M-CSF (50 ng/mL) and RANKL $\left(100 \mathrm{ng} / \mathrm{mL}\right.$ ) for $14 \mathrm{~d}$. (B) The number of TRAP-positive osteoclasts from samples. Mean \pm SD. $n=3$. ${ }^{* *} P<0.01$. (C) Human osteoclast precursor survival by MTT assay: PBMCs in the presence of M-CSF and RANKL for $5 \mathrm{~d}$, then incubated on vehicle or with QOA-8a for $24 \mathrm{~h}$. (D) PBMCs in the presence of M-CSF and RANKL for $5 \mathrm{~d}$. The osteoclast precursors were pretreated with vehicle or with $5 \mu \mathrm{mol} / \mathrm{L}$ QOA-8a and stimulated with RANKL in a time-course experiment, and Western blot analysis of ERK1/2, JNK, p38, p65, IkB $\alpha$ activating phosphorylation. (E-H) hFOB1.19 cells incubated on vehicle or with QOA-8a for $24 \mathrm{~h}$, ELISA assay for protein expression of ALP, BMP-2 and OPG. (I) hFOB1.19 cells were treated with $5 \mu \mathrm{mol} / \mathrm{L}$ QOA-8a in a time-course experiment, and Western blot analysis of ERK1/2, JNK, p38 activating phosphorylation.

postmenopausal women due to the dramatic estrogen withdrawal, an OVX rat model was used because it replicates several key features of the estrogen-deficient adult human skeleton in postmenopausal osteoporosis, such as high rates of bone remodeling, perforation of trabecular plates, loss of trabecular elements of cancellous bone, and thinning and porosity of cortical bone ${ }^{[33]}$. Thus, we used the ovariectomyinduced model of postmenopausal osteoporosis to test the dual functions of QOA-8a. The bone anabolic and antiresorptive effects of QOA-8a were observed by increased trabecular microarchitecture, degraded serum levels of bone resorption markers and elevated levels of bone formation markers, different from the antiresorptive agents. Moreover, the cortical and cancellous bone adjacent to bone marrow were most responsive to QOA-8a treatment; the dosage of 1 $\mathrm{mg} \cdot \mathrm{kg}^{-1} \cdot \mathrm{d}^{-1}$ showed the most significant effects. Alendronate sodium decreased endosteal circumference but had little effect on periosteal circumference, which may relate to a full antiresorptive effect mediated largely through bone remodeling. In contrast, QOA-8a treatment not only decreased endosteal circumference but also increased periosteal circumference, and $5 \mathrm{mg} \cdot \mathrm{kg}^{-1} \cdot \mathrm{d}^{-1}$ showed the most significant effects, which demonstrated that QOA-8a has both antiresorptive and anabolic properties.

The receptor activator of NF-KB (RANK) and its ligand RANKL have been identified as essential factors involved in osteoclast development and bone remodeling ${ }^{[34]}$. The binding of RANKL to its receptor RANK leads to a cascade of intracellular events, including recruitment of receptorassociated factor (TRAFs) ${ }^{[35]}$; activation of the transcription factor nuclear factor- $\mathrm{KB}(\mathrm{NF}-\mathrm{kB})^{[36]}$; activation of the mitogenactivated protein kinase (MAPK $)^{[37]}$ pathways including extracellular signal-regulated kinase 1/2 (ERK1/2), p38MAPK and Jun-N-terminal kinase (JNK); and up-regulation of $\mathrm{c}-\mathrm{Fos}^{[38]}$ and the nuclear factor of activated $\mathrm{T}$ cells $\mathrm{c} 1$ $(\text { NFATc1 })^{[30]}$, two transcription factors needed for osteoclast differentiation. To gain insight into the mechanisms underlying the effect of QOA-8a on inhibiting osteoclast differentiation, we explored the activation status of the above mentioned key signals in the signaling pathways involved in the regulation of this osteoclastogenesis. The results suggested that c-Fos seems to be one of the target genes for the QOA-8a mediated inhibitory effect on osteoclast formation, and QOA-8a inhibits osteoclastogenesis mainly through the ERK/ 
$\mathrm{c}-$ Fos signaling pathway. NF- $\mathrm{kB}$ controls the differentiation or activity of the major skeletal cell types, including osteoclasts and osteoblasts ${ }^{[36,39]}$ Among all NF-KB signals, p65 plays an important role in the osteoclasts, and mice lacking p65 globally showed fewer osteoclasts at baseline and a significantly blunted osteoclastogenic response to RANKL injection ${ }^{[40]}$. Our results confirmed that NF-kB-p65 signaling is also involved in the inhibition of osteoclast differentiation by QOA-8a.

It was recently shown that Janus kinase (JAK)-signal transducer and the activator of the transcription (STAT) signaling pathway play an important role in bone development and metabolism ${ }^{[41]}$. Among all the STATs, STAT3 is probably the most important transcription factor mediating intracellular signaling in osteoblasts, and the lack of STAT3 in osteoblasts may antagonize bone anabolic factors and attenuate the mechanically induced new bone formation ${ }^{[42]}$. We therefore examined whether QOA-8a led to the activation of STAT3 and observed that QOA-8a up-regulated STAT3 phosphorylation in osteoblasts. These results suggested that QOA-8a stimulates osteoblast differentiation through the JNK, ERK/STAT signaling pathway.

Our findings also showed that ERK1/2 seemed to play a key role in the opposite-dual action of QOA-8a; increases in bone mass were observed as a result of pathway activation of ERK1/2 in osteoblasts and decreases in bone loss were noted as a result of pathway inhibition of ERK1/2 in osteoclasts, which was also involved in human osteoclastogenesis and osteoblastogenesis.

OPG is a soluble decoy receptor for RANKL, and it functions to reduce osteoclastogenesis by competitive occupation of the stromal RANKL binding sites for RANK receptors on precursor and later-stage osteoclasts; high RANKL expression promotes osteoclastogenesis and survival, whereas high OPG results in low osteoclastogenesis and osteoclast apopto$\operatorname{sis}^{[4]}$. The results demonstrated that OPG expression level was significantly up-regulated, and the RANKL level was downregulated in the mouse calvarial osteoblastic cells by QOA-8a, indicating that QOA-8a impacted mouse osteoblasts to control osteoclast development and resorption through RANKL and OPG output. However, QOA-8a had no apparent effect on the protein expression of OPG in the human osteoblast. However, accumulating evidence indicates that BMP-2, synthesized and released by osteoblasts and stored in the bone matrix, is a major and indispensable player during bone formation and bone repair ${ }^{[43]}$, and the high level of BMP-2 expression might support the idea of anabolic effects of QOA-8a in humans.

For many years, the medical community has been investigating bone-building therapies that actively increase bone mass by stimulating new bone formation together with antiresorptive treatments. However, in the osteoporosis market, no drug has dual effects on stimulating bone formation and inhibiting bone resorption. Across all studies, our findings may provide a new potential frontrunner in anti-osteoporosis drug development. The next challenge in our work is to discover the target protein(s) of QOA-8a and collect long-term data from ongoing studies.

\section{Acknowledgements}

This work was supported by the National Natural Science Foundation of China (№ 21272114, 90913023). We thank the Shanghai Synchrotron Radiation Facility (SSRF) for the microcomputed tomography $(\mu \mathrm{CT})$ analysis of bone. We thank Prof Shigetoshi KADOTA and Dr Feng LI from the University of Toyama, Japan, for the peripheral quantitative computed tomography (pQCT) analysis of bone.

\section{Author contribution}

Jian-xin LI designed and conceived the experiments. Jing WU, Qi SHEN, Wei CUI, Yu-chao ZHANG and Chen-xi LIU performed the cell biology and biochemical experiments. Yu ZHAO, Yue HUAI and Bei-hua BAO contributed to the in vivo experiments and the histomorphometry analysis. Jian-xin LI, Jing WU and Qing JIANG analyzed the data. Jian-xin LI and Jing WU wrote the paper. All authors reviewed, revised and approved the manuscript.

\section{References}

1 Aw D, Thain J, Ali A, Aung T, Chua WM, Sahota O, et al. Predicting fracture risk in osteoporosis: the use of fracture prediction tools in an osteoporosis clinic population. Postgrad Med J 2016; 92: 267-70.

2 Hofbauer LC, Rachner TD. WNT signaling in bone homeostasis and disease: from human mutations to treatments. Lancet 2015; 386: 1116-8.

3 Nikander R, Sievänen H, Heinonen A, Daly RM, Uusi-Rasi K, Kannus P. Targeted exercise against osteoporosis: A systematic review and meta-analysis for optimising bone strength throughout life. BMC Med 2010; 8: 1-16.

4 Johnell 0 , Kanis JA. An estimate of the worldwide prevalence, mortality and disability associated with hip fracture. Osteoporos Int 2004; 15: 897-902.

5 Kanis JA, McCloskey EV, Johansson H, Cooper C, Rizzoli R, Reginster JY. European guidance for the diagnosis and management of osteoporosis in postmenopausal women. Osteoporos Int 2013; 24 : 23-57.

6 Dempster DW. Osteoporosis and the burden of osteoporosis-related fractures. Am J Manag Care 2011; 17: S164-169.

7 General Office of the Ministry of Health of the People's Republic of China. Outline of prevention and treatment of osteoporosis. Capital J Public Health 2011; 5: 146-9.

8 Harsløf T, Langdahl BL. New horizons in osteoporosis therapies. Curr Opin Pharmacol 2016; 28: 38-42.

9 Opar A. Late-stage osteoporosis drugs illustrate challenges in the field. Nat Rev Drug Discov 2009; 8: 757-8.

10 McClung MR. Emerging therapies for osteoporosis. Endocrinol Metab 2015; 30: 429-35.

11 Deal C. Potential new drug targets for osteoporosis. Nat Rev Rheumatol 2009; 5: 20-7.

12 Garber K. Two pioneering osteoporosis drugs finally approach approval. Nat Rev Drug Discov 2016; 15: 445-6.

13 Deeks ED, Dhillon S. Spotlight on strontium ranelate in postmenopausal osteoporosis. Drugs Aging 2010; 27: 771-3.

14 Ma YFL, Zeng QQ, Porras LL, Harvey A. Moore TL, Shelbourn TL, et al. Teriparatide [rhPTH (1-34)], but not strontium ranelate, demonstrated bone anabolic efficacy in mature, osteopenic, ovariectomized rats. Endocrinology 2011; 152: 1767-78.

15 Fuchs RK, Allen MR, Condon KW, Reinwald S, Miller LM, McClenathan 
D, et al. Strontium ranelate does not stimulate bone formation in ovariectomized rats. Osteoporos Int 2008; 19: 1331-41.

16 Gupta A, March L. Treating osteoporosis. Aust Prescr 2016; 39: 40-6.

17 Baron R, Ferrari S, Russell RG. Denosumab and bisphosphonates: different mechanisms of action and effects. Bone 2011; 48: 677-92.

18 Koch L. Therapy: Raising BMD-two drugs are better than one. Nat Rev Endocrinol 2013; 9: 442.

19 Herrero S, Pico Y. Treatments for post-menopausal osteoporotic women, what's new? How can we manage long-term treatment? Eur J Pharmacol 2016; 779: 8-21.

20 llić D, Bukumirić Z, Janković S. Drug-related problems in patients with osteoporosis. Vojnosanit Pregl 2016; 73: 261-5.

21 Newman DJ, Cragg GM. Natural products as sources of new drugs over the 30 years from 1981 to 2010. J Nat Prod 2012; 75: 311-35.

22 Li JX, Hareyama T, Tezuka Y, Zhang Y, Miyahara T, Kadota S. Five new oleanolic acid glycosides from Achyranthes bidentata with inhibitory activity on osteoclast formation. Planta Med 2005; 71: 673-9.

23 He CC, Hui RR, Tezuka Y, Kadota K, Li JX. Osteoprotective effect of extract from Achyranthes bidentata in ovariectomized rats. J Ethnopharmacol 2010; 127: 229-34.

24 Li JF, Zhao Y, Cai MM, Li XF, Li JX. Synthesis and evaluation of a novel series of heterocyclic oleanolic acid derivatives with anti-osteoclast formation activity. Eur J Med Chem 2009; 44: 2796-806.

25 Zhao Y, Huai Y, Jin J, Geng M, Li JX. Quinoxaline derivative of oleanolic acid (QOA-8a) inhibits osteocalstic bone resorption and prevents ovariectomy induced bone loss. Menopause 2011; 18: 690-7.

26 Nadri S, Soleimani M, Hosseni RH, Massumi, M, Atashi A, Izadpanah R. An efficient method for isolation of murine bone marrow mesenchymal stem cells. Int J Dev Biol 2007; 51: 723-9.

27 Mundy G, Garrett R, Harris S, Chan J, Chen D, Rossini G, et al. Stimulation of bone formation in vitro and in rodents by statins. Science 1999; 286: 1946-9.

28 Clines GA, Mohammad KS, Bao YD, Stephens OW, Suva LJ, Shaughnessy JD, et al. Dickkopf homolog 1 mediates endothelin-1stimulated new bone formation. Mol Endocrinol 2007; 21: 486-98.

29 Katy JLB, Andrew H, Les I, Marc C H, Kristine EE, Steven R, et al. The Potential value of monitoring bone turnover markers among women on alendronate. J Bone Miner Res 2012; 27: 195-201.

30 Kharkwal G, Chandra V, Fatima I, Dwivedi A. Ormeloxifene inhibits osteoclast differentiation in parallel to downregulating RANKL-induced
ROS generation and suppressing the activation of ERK and JNK in murine RAW264.7 cells. J Mol Endocrinol 2012; 48: 261-70.

31 Ikeda F, Nishimura R, Matsubara T, Tanaka S, Inoue J, Reddy SV, et al. Critical roles of c-Jun signaling in regulation of NFAT family and RANKL regulated osteoclast differentiation. J Clin Invest 2004; 114: 475-84.

32 Robling AG, Castillo AB, Turner $\mathrm{CH}$. Biomechanical and molecular regulation of bone remodeling. Annu Rev Biomed Eng 2006; 8: 45598.

33 Tian XY, Jee WSS, Li XD, Paszty C, Ke HZ, Sclerostin antibody increases bone mass by stimulating bone formation and inhibiting bone resorption in a hindlimb-immobilization rat model. Bone 2011; 48: 197-201.

34 Boyle WJ, Simonet WS, Lacey DL. Osteoclast differentiation and activation. Nature 2003; 423: 337-42.

35 Kim N, Kadono Y, Takami M, Lee J, Lee SH, Okada F, et al. Osteoclast differentiation independent of the TRANCE-RANKTRAF6 axis. J Exp Med 2005; 202: 589-95.

36 Novack DV. Role of NF-KB in the skeleton. Cell Res 2011; 21: 16982.

37 Wei ZF, Tong B, Xia YF, Lu Q, Chou GX, Wang ZT, et al. Norisoboldine suppresses osteoclast differentiation through preventing the accumulation of TRAF6-TAK1 complexes and activation of MAPKs/NFkB/c-Fos/NFATc1 pathways. PLoS One 2013; 8: e59171.

38 Grigoriadis AE, Wang ZQ, Cecchini MG, Hofstetter W, Felix R, Fleisch $\mathrm{HA}$, et al. C-Fos: a key regulator of osteoclast-macrophage lineage determination and bone remodeling. Science 1994; 266: 443-8.

39 Boyce BF, Yao ZQ, Xing LP, Functions of nuclear factor KB in bone. Ann NY Acad Sci 2010; 1192: 367-75.

40 Vaira S, Alhawagri M, Anwisye I, Kitaura H, Faccio R, Novack DV. ReIA/ p65 promotes osteoclast differentiation by blocking a RANKL-induced apoptotic JNK pathway in mice. J Clin Invest 2008; 118: 2088-97.

41 Li JL. JAK-STAT and bone metabolism. JAK-STAT 2013; 2: e23930.

42 Zhou H, Newnum AB, Martin JR, Li P, Nelson MT, Moh A, et al. Osteoblast/osteocyte-specific inactivation of Stat3 decreases loaddriven bone formation and accumulates reactive oxygen species. Bone 2011; 49: 404-11.

43 Tsuji K, Bandyopadhyay A, Harfe BD, Cox K, Kakar S, Gerstenfeld L, et al. BMP2 activity, although dispensable for bone formation, is required for the initiation of fracture healing. Nat Genet 2006; 38: 1424-9. 